\title{
Entretien de Claude Lévi-Strauss
}

avec Patrick Menget, Jean-Marie Benoist et Pierre Berès

Philippe Erikson

\section{(2) OpenEdition}

Journals

Édition électronique

URL : https://journals.openedition.org/jsa/17132

DOI : 10.4000/jsa. 17132

ISSN : 1957-7842

\section{Éditeur}

Société des américanistes

Édition imprimée

Date de publication : 20 décembre 2019

Pagination : 15-42

ISSN : 0037-9174

\section{Référence électronique}

Philippe Erikson, «Entretien de Claude Lévi-Strauss », Journal de la Société des américanistes [En ligne], 105-2 | 2019, mis en ligne le 20 décembre 2019, consulté le 03 septembre 2022. URL : http:// journals.openedition.org/jsa/17132 ; DOI : https://doi.org/10.4000/jsa.17132 


\title{
Entretien de Claude Lévi-Strauss
}

\author{
avec Patrick Menget, Jean-Marie Benoist et Pierre Berès
}

\author{
texte édité par Philippe ERIKSON \\ avec la collaboration de Dominique Michelet*
}

En juin 2008, lors d'un colloque organisé au musée du quai Branly pour célébrer le centenaire de Claude Lévi-Strauss, un des intervenants attisa la curiosité de l'auditoire en citant des extraits d'un entretien qui, bien que réalisé trente années auparavant, était demeuré inédit (Menget 2008). Les bandes magnétiques de l'enregistrement original avaient certes disparu, mais Menget conservait un exemplaire tapuscrit de la transcription, qu'il eut la générosité de nous confier. Telle est la source des larges extraits qui suivent, soigneusement édités au cours de l'été suivant.

L'entretien avait eu lieu en 1978, à l'initiative de l'éditeur et bibliophile Pierre Berès [PB] (1913-2008), qui projetait de réaliser un ouvrage d'entretiens avec Claude Lévi-Strauss [CLS]. Il l'avait rencontré en compagnie de l'ethnologue Patrick Menget [PM] (1942-2019) et du philosophe Jean-Marie Benoist [JMB] (1942-1990), l'un et l'autre fins connaisseurs de son œuvre. Le premier venait de soutenir, sous sa direction, une thèse de doctorat (Menget 1977), tandis que le second avait conçu et introduit cette même année l'édition du séminaire interdisciplinaire qu'il avait aidé Lévi-Strauss à organiser au Collège de France durant l'année 1974-1975 (Benoist 1977). L'ambition de Pierre Berès était de faire dialoguer de brillants jeunes chercheurs (sur un mode qu'il qualifiait de « socratique ») avec l'éminent anthropologue, espérant obtenir de lui des commentaires sur des thématiques nouvelles ne concernant ni sa personne, ni son œuvre. Lévi-Strauss se prêta de bonne grâce à l'exercice, du moins pour un entretien préliminaire. Toutefois, se déclarant peu convaincu de la pertinence de cet exercice de maïeutique, et craignant par ailleurs que ce projet ne le détourne trop de son programme de travail, il ne donna pas de suite à ce premier dialogue et l'ouvrage ne vit jamais le jour.

Trente ans plus tard, la rédaction du Journal de la Société des américanistes avait envisagé d'annexer cet inédit aux actes du colloque du quai Branly évoqué

* P. Erikson: LESC (UMR 7186), université Paris Nanterre [erikson@parisnanterre.fr]; D. Michelet: ArchAm (UMR 8096), CNRS [d.m.a.michelet@gmail.com]. 
ci-dessus (Erikson 2008). Lévi-Strauss, à qui le manuscrit avait été envoyé pour validation, s'était toutefois déclaré trop affaibli pour relire le texte avant d'en autoriser la diffusion. Une lettre émouvante datée du 15 octobre 2008 et adressée au directeur de la rédaction de l'époque le formulait en ces termes: «Gravement atteint par l'âge et par la maladie, il ne faut plus rien attendre de moi et rien me demander. Mon seul souvenir de la soirée Berès est celui d'une tentative ratée $[. .$.$] et je ne suis plus en état de le réécrire quand il le$ faudrait. » En dépit de l'intérêt intrinsèque de l'entretien, Lévi-Strauss était donc réticent à le voir paraître de son vivant, et la rédaction avait alors naturellement respecté sa volonté. Les années ayant passé, et le risque de le froisser ayant malheureusement disparu avec lui, il semble aujourd'hui opportun de sortir des archives cet entretien qui éclaire un moment de son œuvre. Nous profiterons également de l'occasion pour transmettre aux intéressés les remerciements différés, mais d'autant plus touchants, du grand homme qui, dans une lettre datée du 30 novembre 2008, avait enjoint à l'organisateur du colloque en son honneur de se faire « si l'occasion s'en présente, [son] interprète pour remercier tous les participants $»$.

En conclusion de l'entretien retranscrit ci-dessous ${ }^{1}$, Lévi-Strauss fait remarquer qu' « il y a des objets qui sont des preuves, des témoignages, d'autres qui n'en sont pas ». À l'évidence, en dépit de son caractère inabouti, ce texte relève de la première catégorie. En cette année $2019^{2}$ et en accord avec Monique LéviStrauss, la rédaction du Journal de la Société des américanistes a jugé utile de diffuser ce témoignage pour honorer, à l'occasion du dixième anniversaire de sa disparition, la mémoire du père fondateur du structuralisme en anthropologie.

\title{
Références citées
}

\author{
BENOIST Jean-Marie \\ 1977 «Facettes de l'identité », in Claude Lévi-Strauss (éd.), L'Identité. Séminaire \\ dirigé par Claude Lévi-Strauss professeur au collège de France 1974-1975, \\ Grasset, Paris, p. 13-23.
}

1.... et dont la forme a été respectée même en cas d'anacoluthe.

2. Par une tragique coïncidence, le hasard fait également de cette année celle de la perte de Patrick Menget, qui fut le mentor de bien des américanistes de ma génération. Quelques semaines avant son décès, Menget m'avait encouragé à publier cet entretien, dont il était alors le dernier protagoniste encore en vie. Il faisait notamment valoir l'intérêt, pour l'histoire de la discipline, de certains de ses passages concernant Durkheim. Hommage d'un maître au maître de son maître... Simultanément à celle de la figure majeure de l'anthropologie de ce pays (Lévi-Strauss), que cette parution soit donc aussi l'occasion de saluer la mémoire de l'un des plus grands sociologues qui l'aient inspiré (Durkheim) et de l'un des plus remarquables ethnologues qu'il ait formés (Menget). Une lignée qui a balisé le chemin des générations précédentes et restera longtemps encore source d'inspiration pour les suivantes. 
ERIKSON Philippe (éd.)

2008 «L'anthropologie structurale? Voyez-vous... it's anthropology at its best », dans « Hommage à Claude Lévi-Strauss », Journal de la Société des américanistes, 94 (2), p. 9-67.

Menget Patrick

1977 Au nom des autres. Classification des relations sociales chez les Txicão du Haut-Xingu (Brésil), thèse de doctorat de troisième cycle, École pratique des hautes études ( $\mathrm{VI}^{\mathrm{e}}$ section), Paris.

2008 « Kinship theory after Lévi-Strauss’s Elementary Structures », Journal de la Société des américanistes, 94 (2), p. 29-37.

\section{Annexe \\ Transcription de l'entretien}

[PB, JMB et PM débattaient entre eux de l'œuvre de Lévi-Strauss quand ce dernier entra. PM lui pose alors une première question dans le prolongement direct de leur conversation.]

PM : Il existe un terme en philosophie bouddhiste, un terme sanscrit, que les traducteurs traduisent alternativement par « vacuité » et « relativité ». Peut-on parler d'une « rencontre » entre la philosophie du structuralisme et celle du bouddhisme, qui refuse l'être?

CLS : Si j'étais obligé d'avoir une religion, je serais bouddhiste. C'est la seule qui ne m'ait pas hérissé quand j'étais en contact avec elle... La religion de l'Inde m’a révulsé.

PM: Est-ce que toutes les manifestations cultuelles ne vous révulsent pas un peu?

CLS : Pas le bouddhisme.

JMB : Est-ce que la philosophie de l'Inde rencontre plus de sympathie chez vous?

CLS : Il y a chez Montherlant une page tout à fait étonnante où il fait une comparaison entre l'esprit juif et l'esprit hindou. Cela me paraît une vérité extraordinaire. C'est dans des carnets, dans des notes...

[Ici s'intercale un passage sur la Colombie britannique qui n'a pas été transcrit.]

PM: Il existe toujours, en Colombie britannique, un savoir traditionnel. Ça inclut la mythologie?

CLS : Oui. Lorsque je suis allé au Canada, je venais de terminer un livre sur un masque. Je crois que je connaissais à fond toute la littérature sur ce masque et toutes les variantes du mythe d'origine. J'ai rencontré un chef indien de la région du masque. Il m'a donné sa version du mythe. Elle était très différente de toutes les versions recueillies... Ce sont des gens très imbus de leur savoir...

PB : Sur quoi portaient principalement les différences?

CLS : D'abord sur le lieu. C'est un masque qui a été pêché dans un lac; ce n'est pas le même lac. Il y a deux sœurs, au lieu d'une sœur et un frère... Ça rentre très 
bien, c'est très facile de savoir où est sa place dans la série... C'est un personnage tout à fait authentique, détenteur de ses traditions. On se rend compte quand on est sur le terrain que les informations dont nous disposons sont des mailles lâches entre lesquelles il y a des tas de choses qui ont passé...

PB: Comment se présentent les Indiens? Quelle est leur proximité avec la vie quotidienne, les drugstores, etc.?

PM: Vous observez dans leurs récits, dans leurs propos, dans leurs savoirs archaïques, l'intégration peu à peu, ou la greffe, de ces éléments modernes. Est-ce qu'il y a une contamination par des thèmes?

CLS : Ils sont complètement modernisés, mais il y a deux secteurs. Un jeune Indien, champion de hockey, retournait chez lui pour être sculpteur; il est obligé d'apprendre la langue, mais la raison pour laquelle il veut être sculpteur c'est que, quand on vend une sculpture, il n'y a pas d'impôts à payer. C'est une très bonne affaire. Il me raconte comment il a un rôle spécial dans les cérémonies. Son rôle, cela consiste à faire une prestidigitation avec du feu, à faire apparaître et disparaître $\mathrm{du}$ feu. Quand il était enfant, ça lui paraissait extraordinaire...

JMB : Il y a une acception de la survivance rituelle qui côtoierait plutôt les thèmes modernes.

CLS : Il y a une renaissance rituelle, il y a des cérémonies qu'il n'y avait plus qui ont été célébrées cette année [1978].

JMB : Donc les énoncés ne se modifient pas du fait qu'ils sont conditionnés, par la télévision par exemple.

CLS : Mais si, ça modifie profondément; en 1973 j'ai été admis par faveur très spéciale à assister à un rituel d'initiation. La fonction d'initiation a changé. Autrefois, c'était pour acquérir un statut à la fois social et surnaturel; maintenant, c'est une espèce de thérapeutique. On leur impose une initiation pour les réintégrer, il s'agit de les attirer là où ils doivent rester.

PM: Le déplacement de certaines fonctions comme ce rite d'initiation, est-ce que vous le caractériseriez comme un bricolage?

CLS : Ce serait abuser du terme. C'est le point d'impact d'un rituel très bien constitué qui s'est déplacé. Le thème n'a pas varié. Les gens sont toujours en quête d'un esprit gardien. Quand ils ont trouvé l'esprit gardien, ils chantent le chant que l'esprit gardien leur a révélé, ils dansent la danse que l'esprit gardien leur a enseignée.

$\mathrm{PB}$ : Mon ambition serait d'arriver à produire un livre qui ne vous donnerait pas de mal, dans lequel vous exposeriez un certain nombre de choses en dehors de celles que vous avez le talent, ou le génie, ou l'art d'exposer vous-même. Une de mes préoccupations aujourd'hui, c'est d'essayer de vous poser des questions ou, plutôt, de vous faire dire des choses vous-même qui ne seraient pas du tout ni une suite, encore moins une redite, de prises de position déjà connues, mais qui rendraient compte d'autres investigations... Je ne peux pas vous mettre sur la voie... : votre position de philosophe et d'autre part les motivations de cette position. Au terme 
des Mythologiques, avant les Mythologiques, nous sommes tous très curieux de ce qu'il y a en vous.

CLS : Je suis venu parce que je ne voulais pas faire la mauvaise tête, mais cela ne marchera pas, parce que je n'ai pas le goût des dialogues. Ma tendance constante, dans le dialogue, c'est de me défiler, de me rétracter, de trouver un moyen d'échapper au dialogue; ça ne me stimule pas du tout... Je ne pense pas en conversation. Pour penser, il faut que je me mette à écrire.

PM: Vous êtes un conférencier remarquable; à chaque séance, on éprouvait pour vous une anxiété, on se disait: «Va-t-il ramasser les fils? » et effectivement, à la fin de la séance, tous les fils étaient ramassés.

CLS : Mais un cours n'est pas une conversation.

PB : Et Rousseau? C'est quelqu'un qui vous a toujours fasciné. Il me semblait qu'il y avait dans cet intérêt que vous portiez à Rousseau une espèce de similitude d'approche dans laquelle l'anthropologie n'était qu'un des moyens.

CLS : Disons qu'il y a comme cela dans ma biographie un moment où je me suis senti faire de l'identification à Rousseau et, à ce moment-là, je l'ai ressenti de façon très aiguë et très vive. C'est passé, c'est éloigné.

PB: On ne peut pas évoquer les états d'âme de cette identification?

CLS : Là, je vous dirai : je ne me rappelle pas, j'oublie. J'ai déjà écrit à ce propos à plusieurs reprises, mais l'identité personnelle me manque totalement. J'ai l'impression qu'on est le lieu où des choses se passent, mais une fois qu'elles ont cessé de se passer, c'est fini.

JMB : Ce n'est pas tant une identification personnelle avec Rousseau que la rencontre avec les textes - Discours sur l'origine de l'inégalité ou Du contrat social - qui vous ont semblé peut-être à un moment opérer d'une façon assez convergente avec le projet anthropologique que vous développiez.

CLS : Peut-être même pas. C'est d'avoir retrouvé une espèce de perspective sur une société autre où je me suis senti dans la même optique qu'avait Rousseau... Tout cela n'est plus présent. C'est il y a vingt ans, ce n'est plus moi, c'est quelqu'un d'autre.

PB : Ce que vous étiez à ce moment-là, on serait bien content de le savoir dans la mesure où vous ne nous l'avez pas dit. Ce que vous étiez à New York en 1941, par exemple.

CLS : Je peux vous donner un bon exemple: ma mère a retrouvé au mois de juillet dans une armoire de sa maison de campagne toutes les lettres que j'écrivais de New York en 1941-1942 et j'ai passé plusieurs jours à relire ces lettres. Pour moi, c'étaient des révélations, je n'avais pas l'ombre d'un souvenir de tout ce que je raconte dedans. J'avais commencé à écrire les Structures ... ${ }^{3}$ et j'avais commencé à m'intéresser à la formation logico-mathématique des systèmes de parenté, avant de rencontrer Jakobson. Tout cela, je ne le savais plus.

3. Structures... pour Les structures élémentaires de la parenté. N. B. Toutes les notes de l'annexe sont de la rédaction. 
PB : Comment avez-vous fait vos recréations à partir de lui?

CLS : Quand il m'a appris ce qu'était la linguistique structurale, j'ai tout à coup reconnu ce que j'avais envie de faire, mais ce qui est certain, c'est que j'ai fait tout à fait autre chose. En quoi cela diffère, je n'ai jamais cherché à le savoir.

PB: Vous l'avez toujours su.

CLS : Non, car pour savoir ça, il faudrait s'appliquer à répondre aux critiques. C'est quelque chose que je ne peux pas faire.

PM: Vous voyez votre vie scientifique comme une suite de moments sans liens que vous oubliez au fur et à mesure. Or, pour les gens qui vous lisent, il y a quand même une très grande cohérence. Vous ne vous voyez pas du tout comme on vous voit. En particulier en ce qui concerne ce que vous n'explicitez pas dans votre philosophie. C'est-à-dire que, étant ethnologue savant, vous avez forcément une épistémologie.

CLS : Vague. Est-ce qu'il y a des choses à expliciter?

$\mathrm{JMB}$ : Votre polémique avec l'existentialisme a tout de même correspondu à une élaboration philosophique ou épistémologique à partir de vos travaux anthropologiques et toujours en contact avec elle. Je pense à des passages de La Pensée sauvage ou également des thèses sur l'histoire: « Histoire et dialectique ». Cela correspond tout de même à des positions épistémologiques qui ne sont pas seulement des réfutations, des boucliers dans les polémiques. Elles restent là dans la matière des mots. Elles continuent à fonctionner pour les lecteurs que nous sommes.

CLS : C'est paradoxal, la cause d'un malentendu constant entre l'auteur et ses lecteurs. Il y a des gens qui ouvrent aujourd'hui les Structures... Pour eux, c'est nouveau, ils m'écrivent... Je m'en fiche de ce que j'ai écrit il y a trente ans.

JMB: Vous ne vous voyez pas du tout dans une séquence par rapport à des écrits actuels ou futurs? Vous vous refusez à poser un rapport de conditionnement, d'approximation et même d'auto-réfutation entre ces divers écrits, entre ces divers moments?

CLS : Non, parce que, pour le faire, il faudrait justement que j'aie le sentiment que j'ai une continuité de pensée et de personne à maintenir, à défendre, à justifier, alors que c'est un sentiment qui me manque.

JMB : Dans cette vision de la succession des phases de votre travail même se reflète un peu votre méfiance de la téléologie historique, de toute finalité, de tout projet qui concernerait l'histoire générale de l'humanité. Je me souviens d'un passage dans lequel vous discutez l'existence d'une histoire à la Hegel... Est-ce qu'il y aurait un rapport? Est-ce que ce serait même une sorte d'indiscrétion épistémologique de pensée? Est-ce qu'il y aurait un rapport entre votre refus de téléologie dans l'avenir de votre œuvre et ce refus de la macro-téléologie: est-ce qu'il y a un rapport?

CLS : Probablement.

PB: Vous disiez tout à l'heure que vous aviez été très actif dans le parti socialiste. Vous n'avez pas produit d'écrits politiques?

CLS : Si, pas mal d'articles. Il y a eu un livre auquel j'ai participé qui s'appelait Révolution constructive. 
PB : Quelle est l'ontologie sur laquelle vous vous appuyiez?

CLS : Ma formation politique, je la devais entièrement au parti ouvrier belge dont j'ai été une sorte de pupille à un moment. Ce petit groupe s'imaginait qu'il était possible de construire la société socialiste à l'intérieur de la société capitaliste, par des coopératives, des syndicats, etc., que cela grossirait. On ne pouvait rien imaginer de plus idiot. Je ne vois ni l'avènement d'une société socialiste, ni la fin d'une société capitaliste. Je suis complètement désintéressé de ces problèmes, je ne crois plus que la politique est pensable.

JMB : Est-ce que vous pensez que notre société est pensable?

CLS : Non. Il y a des choses qui sont pensables. Par penser notre société, je veux dire être capable de maîtriser par la pensée la multiplicité de variables qui sont à l'œuvre à l'intérieur de notre société, ce n'est pas une réaction à l'extérieur de cet ensemble de valeurs morales... Je n'ai pas du tout fait un détour anthropologique pour m'interroger sur la société, j'ai fait un détour anthropologique parce que cela m'ennuyait d'enseigner la philosophie. Parce qu'il se trouvait que Nizan avait épousé une de mes cousines et qu'il m'a dit: «Quand on est philosophe, on peut filer du côté de l'ethnologie. » Je me suis dit: « Pourquoi pas? »; c'était beaucoup plus par goût de l'exotisme et du camping que pour m'interroger sur la société.

JMB : Je ne crois pas qu'on part à l'autre bout du monde pour s'interroger sur sa société. C'est un résultat possible au retour, quand on est parti.

CLS : On peut partir parce qu'on se pose des problèmes sociologiques. Ce n'était pas du tout mon cas.

PB : Ayant fabriqué une œuvre de cette importance, vous êtes revenu trop chargé de problèmes personnels du savant que vous êtes devenu à ce moment-là, ce qui a probablement masqué l'interrogation que, simple citoyen, vous auriez conservée?

CLS : Je crois que vous poétisez les choses.

PB : Ce serait une explication du refus que vous opposez. Il fallait que ce travail fût suffisamment intense pour que les autres questions ne se posent plus à vous...

CLS : Je dirais que c'est une évolution normale de faire du militantisme quand on est jeune et de s'en désintéresser plus tard. C'est arrivé à des milliers de personnes.

JMB : Il y a des engagements plus prioritaires : écrire, travailler, rassembler, rendre justice à cette matière que vous aviez collectée...

CLS : Il y a tellement de contingence qui rentre là-dedans. Je faisais activement de la politique quand je suis parti au Brésil. C'était la veille de la guerre, j'ai filé aux États-Unis. J'ai reçu des propositions pour rejoindre de Gaulle à Londres. Si je l'avais fait, probablement que j'aurais été dans la politique au lendemain de la Libération. Je ne l'ai pas fait. Pourquoi? Je me plaisais beaucoup à New York, à n'avoir aucune orientation.

PB : Quels étaient vos rapports avec Breton à ce moment-là?

CLS : Fort intimes. Nous nous étions beaucoup vus sur le même bateau qui nous amenait, puis nous nous sommes perdus à la Martinique parce que je suis parti sur un bateau différent du sien. J'ai retrouvé dans des lettres le récit de mes retrouvailles avec Breton sous la forme suivante: nous nous étions téléphoné, 
il devait venir me voir et puis il n'était pas là; puis j'ai reçu de lui un coup de téléphone angoissé d'un café; nous avons repris contact. On se voyait énormément, plusieurs fois par semaine.

PB : Comment est-ce que vous réagissiez à cette papauté?

CLS : Je ne faisais pas vraiment partie du groupe. On n'a jamais considéré que j'étais engagé par ce que faisait le groupe. Il y avait Duchamp, Tanguy, Max Ernst, Masson, sur quoi Breton régnait.

PB : Qu'est-ce qu'il vous apportait, est-ce que son écriture vous impressionnait?

CLS : J'avais déjà lu Breton avant, j'avais beaucoup admiré Breton. C'était pour moi une espèce de personnage mythique.

PB: Je soupçonne Breton de s'être assez peu intéressé aux autres à New York.

CLS : Il était très gentil. J'ai encore des lettres de lui du bateau. Ce n'est pas publiable. Nous échangions des petites lettres, c'était sur la question de savoir si l'originalité absolue était un gage de beauté. C'était une petite correspondance. Probablement que j'avais dit non. Il m'a énormément appris, nous courrions les antiquaires. Il y avait ça, il y avait les jeux de la vérité, c'était très affriolant pour moi car je n'avais jamais connu ça, une telle liberté, sinon de mœurs car ils étaient très puritains en vérité. J'y étais en spectateur... Jakobson et Breton se voyaient à travers moi. Moi, je n'avais jamais été dans des milieux artistiques ou littéraires d'avant-garde, tandis que Jakobson y était allé en Russie: les peintres russes...

PB : Comment expliquez-vous pourquoi vous avez oublié tant de choses, même de votre correspondance, alors que vous êtes un des hommes qui ont la plus belle mémoire?

CLS : Tout cela est fait à coups de fiches. Sans cela, je ne me rappellerais pas. $\mathrm{Ou}$, plus exactement, je me rappelle pendant que je le fais, dès que c'est fini, c'est oublié...

PB : Il y a sept ou huit ans j'avais commencé la collection « Miroir de l'art». Un mot de Françoise Cachin m'avait fait entendre que vous prépariez un Rousseau; que vous m'en auriez donné un morceau. Vous avez donc pensé cela?

CLS : J'ai toujours pensé qu'il pouvait y avoir une esthétique de Rousseau.

PB : C'est quoi, l'esthétique de Rousseau?

CLS : Il y a quelque chose que je me rappelle de façon fugitive. Dans l'Essai sur l'origine des langues, il invente par anticipation ce que c'est que la peinture non figurative pour s'en servir à des fins critiques. Il y a, d'autre part, tout l'antagonisme entre Rousseau et Rameau. Cela pose tout un problème parce que le formaliste, c'est Rameau, et on se sent plutôt du côté de Rousseau. Qu'est-ce que c'était exactement que la position de Rousseau? C'était la position de l'art naîf devant l'art savant. En écoutant Le Devin du village, j'ai toujours été frappé de l'extraordinaire maîtrise qu'il avait de la prosodie du français et qu'au fond, cela se produit deux fois dans l'histoire de la musique. Cela se produit avec Le Devin $d u$ village et, ensuite, avec Pelléas. Mais savoir faire chanter le français, il n'y a que Rousseau et Debussy qui l'ont su. 
JMB : Est-ce que c'est important pour vous, Le Devin du village?

CLS : C'est très réussi. Il y a un disque ${ }^{4}$ ! Le problème c'est que, pour faire quelque chose sur l'esthétique de Rousseau, il faudrait être beaucoup plus calé en musique que je ne le suis.

JMB : Mais vous avez, dans des passages des Mythologiques, montré beaucoup de savoir sur la composition musicale, sur l'analyse.

CLS : Vous savez, ce que j'ai fait pour le Boléro, je n'aurais peut-être pas pu le faire pour autre chose que pour le Boléro. C'était particulièrement facile. J'ai essayé de le faire pour autre chose... J'avais essayé sur la Pastorale; il y a des choses très étranges. On a l'impression qu'on entend des choses qui coulent et, quand on regarde la partition, la manière dont c'est construit métriquement est très compliquée. Je me suis dit que ce serait intéressant de comprendre pourquoi ce n'est pas possible.

PB: Mais les rapports de Rousseau avec les peintres de son temps sont assez maigres $[\ldots]$.

CLS : On voit bien pourquoi il ne s'est pas intéressé à la peinture. Parce que c'était bourgeois, sophistiqué... Pourquoi il a pensé qu'en musique ce n'était pas comme en peinture: il y avait la possibilité de faire quelque chose en musique qu'on ne pouvait pas faire en peinture... probablement cette idée latente que la musique, c'est de plain-pied avec une expression populaire. Les sauvages chantent, dansent, mais ils ne font pas de tableaux, peut-être que c'est une explication...

JMB : La musique est beaucoup plus proche de la fête.

CLS : Cela demanderait qu'on lise tout Rousseau dans cette perspective, qu'on lise toute la correspondance. J'ai écrit comme ça cinq, six passages légers sur Rousseau pour le $250^{\mathrm{e}}$ anniversaire de sa naissance à Genève. Gouhier, avec qui je suis en excellentes relations, m'a écrit un jour: "Tout de même, ce que vous avez écrit là n'est pas vrai. » Il a raison, car il connaît Rousseau mieux que moi. Il a travaillé en érudit sur Rousseau, ce que je n'ai pas fait.

PB: Quelle est la part de rigueur et d'érudition que vous exigez de votre création? Quel est, entre l'artiste et le savant, le mariage que vous faites?

CLS : J'espère être un savant. L'artiste, c'est par-dessus le marché.

PB : Il n'y a jamais bousculade de l'un sur l'autre?

CLS : Très évidemment, constamment. Quand j'ai l'idée d'un article, je vais très au-delà de ce que je suis en droit scientifiquement d'affirmer et alors le travail d'écriture consiste à vérifier, à revenir en arrière sur ce que j'imaginais... Écrire c'est une agonie. Je suis très sensible à l'incantation des idées.

PB: Moi, ce qui m'intéresse, c'est l'avatar de Lévi-Strauss, sa position philosophique.

CLS : Vous voulez que je donne une position philosophique, alors que j'ai constamment écrit que je n'ai pas de position philosophique! Ou alors que j'adapte n'importe laquelle qui m'arrange au moment où...

4. Jean-Jacques Rouseau, Le Devin du village. Danièle Borst, Louis Devos, Philippe Huttenlocher, Orchestre «Le Collegium Academicum », Genève, sous la direction de Robert Dunand, CBS 76716 (1 V), 1978. 
PB : Vous avez beaucoup fréquenté Chomsky?

CLS : Je ne le connais pas.

PB : C'est un homme qui vous intéresse?

CLS : C'est très difficile pour moi. Je lui pardonne mal d'avoir rendu la linguistique ennuyeuse.

JMB : Mais l'aspect transformationnel ne vous intéresse pas? Vous aviez vousmême repéré les systèmes de transformations, vous ne pensez pas qu'entre Jakobson et votre travail?...

CLS : Les systèmes de transformations : il disait qu'il fallait utiliser des transformations à la science de l'homme; c'est ni Chomsky, ni moi qui l'avons inventé, c'est déjà dans un article de Cassirer de 1938.

JMB : Mais le parallèle entre ce que, lui, a fait et ce que vous avez fait ne vous intéresse pas?

CLS : Cela, je crois que c'est très, très superficiel parce que son transformationnisme est purement formel, le mien est sémantique.

JMB : Et l'articulation de l'un à l'autre, même malgré cette différence, ne vous paraît pas être un terrain intéressant?

CLS : On peut dire que, moi aussi, je cherche des structures profondes des mythes, mais ce n'est pas la même chose, cela recouvre des contenus tout à fait différents. Ce qui me gêne dans les structures profondes de Chomsky, c'est que ses structures profondes sont encore des structures linguistiques et donc cela impliquerait que nous pensons en petit-nègre avant de penser dans notre langue, alors que mes structures profondes sont d'une autre nature que les structures superficielles.

JMB : Si on vous dit « générativité », chez vous cela se situe tout à fait différemment, vous êtes dans l'après.

CLS : Les Structures... sont un livre génératif: il s'agit d'engendrer un certain nombre de règles de mariage à partir d'un petit nombre de principes.

PM: Comment avez-vous reçu la critique de Chomsky sur le type de structures dont s'occupent les structuralistes, où Chomsky dit: " Au fond, ce n'est qu'un artifice de la procédure de découverte et cela ne va pas bien loin. » Il me semble surtout, dans sa critique, qu'il s'adressait aux linguistes.

CLS : Je crois que ce que fait la linguistique structurale classique et ce que j'essaie de faire, même, atteint la façon dont on pense. Quand je suis en face des transformations de Chomsky, je ne peux pas croire qu'on pense comme ça. Je suis tout prêt à croire que c'est la manière la plus économique pour essayer de faire fabriquer des structures superficielles par un ordinateur, mais je ne crois pas qu'un esprit humain pense comme un ordinateur.

JMB : C'est la sémantique qui empêche l'esprit humain de penser comme un ordinateur? Quel serait l'obstacle?

CLS : C'est le côté additif de la transformation chomskyenne. On commence par quelque chose et on ajoute quelque chose et on ajoute encore quelque chose. J'ai l'impression que l'esprit travaille par une espèce d'appréhension globale du résultat qui s'affine, et cela fonctionne dans l'autre sens. 
PB: Vous réfutez le microscope? Le microscope n'y verrait pas autre chose.

CLS : Je refuse ce cartésianisme à l'envers. Je ne crois pas qu'on le libère de cette façon-là par des parties qu'on ajoute les unes aux autres et qui finalement donnent un résultat.

PB: Ce qui débouche très vite sur le problème de l'intuition, alors, à quelque degré qu'elle se situe.

CLS : C'est plutôt un côté mécanisme et téléonomie.

JMB : Qui va de pair avec une métaphysique du sujet que vous critiquez d'autre part.

CLS : Je suis tout prêt à reconnaître qu'il [Chomsky] a fait des choses très importantes dans la théorie de la syntaxe... Que la théorie de la syntaxe ait été négligée par les structuralistes classiques... Ou les structures sont profondes et alors elles ne sont pas déjà linguistiques, ou elles sont déjà linguistiques et elles ne sont pas profondes. Piaget a dit des choses très justes là-dessus. Il y a en effet des structures profondes, mais qui ne sont pas proprement linguistiques, qui sont communes à toutes les formes de l'activité symbolique et qui se spécifient en langage. Mais cette idée qu'il y a quelque chose sous le langage et que c'est encore du langage, à mon avis cela ne marche pas, parce qu'alors il y a encore quelque chose dessous et ainsi de suite indéfiniment. Et si, sous les structures profondes, il y a des structures non linguistiques, alors je ne vois pas pourquoi on a besoin des structures profondes.

PM: Chomsky l'a fait probablement parce que c'est commode, c'est simple. C'est un bon mécanisme générateur, cela permet de rendre compte de la syntaxe, en particulier. L'objet de Chomsky n'étant pas au départ de rendre compte de toute l'activité de l'esprit humain, mais de la langue et de la façon dont cela parle, dont cela circule.

CLS : Oui, qu'il ait fabriqué des règles qui sont parfaitement cohérentes et qui permettent de reconstituer un certain nombre de choses, je veux bien... Simplement, je ne peux pas croire que c'est comme ça que cela fonctionne.

PM: Ce n'est pas sa métaphysique qui vous gêne, sa métaphysique du sujet cartésien de l'infinité des possibles?

CLS : Pourquoi est-ce qu'il y a une infinité des possibles? Cela ne me paraît pas sérieux. Pratiquement tout se passe comme si c'était infini, donc de savoir si ça l'est en droit et en fait, c'est une question scholastique, ça devient gênant à partir du moment où on s'en sert pour en faire une métaphysique...

PB : Quelles ont été les réactions de Chomsky par rapport à l'œuvre de Lévi-Strauss?

CLS : Il est très négatif; il en a très peu parlé et, au fond, ce qu'il en a dit, c'est que cela ne l'intéresse pas.

JMB : Il vous voit comme une extension d'une méthode linguistique.

PB: Cette façon de réfuter Chomsky, ou plutôt ce mécanisme, est déjà une prise de position philosophique, finalement, que vous le vouliez ou non.

CLS : Ce n'est pas une réfutation, je ne me le permettrai pas parce que je ne suis pas assez linguiste pour faire la réfutation, c'est pourquoi cela ne m'accroche pas. 
JMB : Est-ce que vous approuvez les gens qui se considèrent comme des hommes de science, mais qui relativisent l'attitude du savant et qui contestent même le projet d'un logos scientifique ou d'une raison scientifique?

CLS : Je serais porté à dire le contraire. Dans notre civilisation, la seule chose qui me paraît mériter le respect et l'estime, c'est la science.

JMB : Dans la mesure où les cultures que vous étudiez en tant qu' anthropologue ne sont pas porteuses de projets scientifiques, vous ne voyez pas ce phénomène d'un regard d'anthropologue. Ce n'est pas un objet pour l'anthropologie, l'anthropologie vise à être une science, mais le fait qu'il y ait une raison scientifique dans une culture particulière n'est pas un objet anthropologique.

CLS : C'est pourquoi je crois à la science.

PM: Vous ne pensez pas que la rationalité scientifique puisse être contestée institutionnellement, c'est-à-dire parce qu'elle est organiquement liée à certaines institutions qu' on peut contester pour d'autres raisons? Donc, du même coup, on rejetterait la rationalité scientifique.

CLS : De la même façon on peut dire qu'il n'y aurait pas d'ethnologie s'il n'y avait pas eu de colonialisme, donc il faut rejeter l'ethnologie. Cela s'est dit.

PB: Il n'y avait pas de colonialisme au temps de Bougainvilliers?

JMB : Si, il y avait quand même des formes d'exploitation, il y avait des formes commerciales.

CLS : Il y avait un embryon de colonialisme, comme il y avait un embryon d'ethnologie, cela s'est tout de même développé de pair, mais l'un à l'abri de l'autre.

JMB : Vous ne pensez pas qu'il y a un terrorisme du discours scientifique pour employer une expression gauchiste, c'est-à-dire que la parole scientifique ne s'impose pas selon des critères rationnels, mais elle s'impose parce qu'elle vient d'une position institutionnelle dans laquelle se trouvent nos savants. C'est l'essentiel de la critique institutionnelle qu'on trouve partout en ce moment aux États-Unis, en France, en URSS également.

CLS : Non, moi je suis imperméable à tout cela. Ce qui m'intéresse, c'est qu'on a découvert le code génétique.

PB: Je voulais vous demander, justement, quels sont vos intérêts ou vos activités avec la science en marche. C'est-à-dire, la découverte physique, même lorsqu'elle est incertaine, vous importe? Est-ce que le code génétique flatte chez vous l'espoir d'une explication plus précise de l'homme, etc.?

CLS : Je lis très attentivement tous les mois le Scientific American. Je ne comprends pas tout.

PB : Et vous êtes plus satisfait d'être Lévi-Strauss, contemporain du code génétique et des travaux de Grothendieck, que si vous étiez Montaigne, contemporain de la zoologie de Watson ${ }^{5}$ ? Vous n'avez pas l'impression que la science, au fond, c'est vous ou Montaigne plutôt que Grothendieck ou Jacques Monod?

CLS : La science, ce sont des physiciens ou des biologistes.

5. PB a possiblement procédé à l'amalgame anachronique du scientifique James Watson - co-inventeur, en 1953, du code génétique et initialement formé à la zoologie - avec un de ses homonymes naturalistes du XVIII ${ }^{\mathrm{e}}$ siècle (PM, comm. pers.). 
PB : Cette institutionalité (ou cette autorité) de la science mène donc des hommes comme chacun de nous - et, ce qui est plus grave, de votre importance - à faire un crédit à quelque chose qui finalement est quelquefois un dogme.

CLS : Il y a, à toutes les époques et dans toutes les sociétés, par rapport à toutes les institutions, des hommes qui ont vu juste et des hommes qui se sont trompés... Il n'y a pas une telle quantité de sociétés qui veulent avoir une science qui soit en accord avec ses dogmes politiques. Enfin, cela s'est vu dans le passé, cela se voit encore aujourd'hui de temps en temps, on ne peut pas dire que cela soit dans le monde entier... J'ai parcouru l'autre jour un article dans Les Temps modernes destiné à montrer que les mathématiques étaient réactionnaires, fonction d'un système institutionnel ${ }^{6}$. J'avoue qu'après avoir lu l'article, j'étais aussi avancé qu'avant. On a même dit que le structuralisme était une philosophie d'un monde technique et technocrate... Du moment que cela arrive dans toutes les sociétés, je ne vois pas pourquoi cela n'arriverait pas dans la nôtre, cela ne crée pas un vice particulier à notre société.

JMB: Le discours scientifique et le statut du savant vous paraissent relever d'une anthropologie, tout de même. Sans contestation politique, est-ce que cela vous paraît un objet d'anthropologie?

CLS : Il y a sûrement une sociologie du savant qui peut être faite. Je ne crois pas que ce soit une anthropologie... Tout cela me parait tellement secondaire et insignifiant par rapport à la démarche de la science.

PB: Donc, vous attendez quoi?

CLS : De comprendre des choses qu'on ne comprenait pas.

PB : C'est intéressant dans votre bouche car vous, justement, vous n'avez besoin de rien de tout ça pour comprendre ce que d'autres n'ont pas compris. Vous n'avez en rien profité des enseignements de la science.

CLS : Mais je dirais : "malheureusement », car si nous étions capables d'en profiter mieux, nous serions capables de faire des choses bien meilleures. Nous ne nous sommes pas encore rejoints dans ce que nous appelons les « sciences humaines », qui manipulent des ombres ou des fantômes.

PB: Mais comme vous, vous êtes l'exemple qu'on peut les manipuler de façon très bonne et très scientifique!

CLS : Non, de façon préscientifique; je dirais volontiers que ce que je fais - et encore je manque de modestie -, au mieux, ce que je fais, cela ressemble à de l'astronomie du temps des Babyloniens... Qui tout de même commençaient à comprendre d'autres choses...

6. CLS fait vraisemblablement allusion ici à un article de Georges Pòr intitulé « Vous n'êtes qu'une donnée de la science », paru dans la livraison de février 1978 de la revue Les Temps Modernes (n' 379, p. 1269-1285). L'auteur y dénonce les usages déshumanisants et aliénants de la statistique auxquels s'abaisserait ce qu'il appelle « la sociologie-chien-de-garde » [sic]. 
JMB : Qu'elle était l'instrument d'un pouvoir...

CLS : Parce qu'en effet, le pouvoir peut s'en servir. Votre comparaison serait juste si le pouvoir se disait: "Lévi-Strauss nous a montré comment les mythes étaient faits, nous avons besoin de mythes pour faire marcher les foules, nous allons donc fabriquer des mythes qui seront susceptibles de paraître comme des vrais mythes et nous pourrons faire marcher les foules. »

JMB : Je ne le pensais pas dans ce sens-là. Je le pensais plutôt dans le sens où je ne connais pas de société où une astronomie ou une astrologie soit née en dehors d'une structure étatique, d'une organisation étatique. Si vous voulez, l'astronomie est liée à l'État.

CLS : L'astronomie n'existerait pas s'il n'y avait pas eu une structure étatique qui a fait aussi le CNRS, des professeurs d'ethnologie... Le pouvoir estime qu'il est bon qu'il y ait des ethnologues, quand ce ne serait, disons, que pour faire un travail de diversion.

JMB : La question à laquelle je voulais en venir c'est: est-ce que votre expérience d'ethnologue et de savant - vos réflexions sur cette société sans État - vous a parfois amené à vous poser des questions sur les sociétés avec État et sur l'État; en particulier, sur notre État?

CLS : On se heurte toujours à cet obstacle qu'on n'est pas devant le même ordre de grandeur et qu'il ne s'agit pas de comprendre ou de préférer des sociétés sans État et des sociétés avec État. Nous sommes devant des sociétés qui sont tellement grosses qu'elles ne peuvent être que des sociétés avec État. On peut se dire qu'on aimerait mieux que les sociétés ne soient pas devenues si grosses, qu'il n'y ait pas tellement d'hommes sur la terre, etc. Mais nous n'avons pas le choix, c'est incomparable.

JMB : Vous pensez que la différence est qualitative? Nous avons affaire à des types de sociétés qui sont incommensurables?

CLS : Je crois que les changements d'échelle ont un rôle; même, du temps où je m'occupais du Conseil international des sciences sociales, j'avais essayé de lancer un grand projet qui n'a pas pu voir le jour parce que les gens n'y comprenaient rien à l'époque, qui était l'influence des changements d'échelle. Quelles sont les choses qui peuvent se passer dans un certain ordre de grandeur et qui ne peuvent pas se passer dans un autre ordre de grandeur?

PM: Au fond, j'objecterais les trouvailles que vous avez vous-même citées, qui sont des trouvailles de démographes et de généticiens sur une sorte d'optimum humain qu'on retrouve dans beaucoup de sociétés primitives sans État et qu'on retrouve à l'état communautaire, donc subordonné, dans nos sociétés complexes. Donc il y a beaucoup de points de comparaison et d'analogie.

CLS : En effet, dans nos sociétés complexes, il y a des niveaux qui fonctionnent dans une certaine mesure très restreinte, mais par certains aspects, comme peuvent fonctionner des sociétés plus simples. C'est pour cela que les études de villages sont tellement intéressantes. 
PB : Mais, dans votre esprit, cela rejoint le mécanicisme de Chomsky en somme, c'est-à-dire que cela rend compte de phénomènes de façon analysable, mais pas réelle.

CLS : Non, au laboratoire il y a des types qui travaillent depuis cinq, six ans dans un village et qui rencontrent des choses très intéressantes. Ce sont des niveaux qui, dans une petite mesure, fonctionnent encore anthropologiquement, si j'ose dire.

JMB : Et vous pensez que la comparaison est à éviter, c'est-à-dire des phénomènes aussi importants dans ces sous-sociétés ou ces niveaux de notre société qui, de plus en plus, apparaissent parfaitement incomparables... Il y a une limite dans la possibilité de comparabilité, c'est-à-dire qu'une science des sociétés devrait être stochastique, être faite de différentes ruptures à différents niveaux.

CLS : Il y a des espèces de sociétés comme il y a des espèces animales.

JMB : Je suis un peu surpris de vous entendre parler d'espèces de sociétés, alors que vous avez été le plus systématiquement opposé à l'évolutionnisme en matière sociale.

CLS : Ce n'est pas une position évolutionniste, je ne dis pas du tout qu'une espèce évolue pour devenir une autre espèce.

JMB : Néanmoins, il existe des rapports entre ces différentes espèces, est-ce que vous pensez que c'est un objet valide d'investigation?

CLS : Bien sûr... c'est-à-dire: qu'est-ce qu'on aurait mieux aimé être? Se pose tout de suite la question très judicieuse de savoir quelle est la position qu'on occuperait dans cette société. Si on était sûr d'occuper la meilleure, alors je dirais n'importe quelle société vaut mieux que la nôtre. Le seul vrai luxe qu'aient jamais eu les hommes, c'est l'esclavage.

PB : Je suis très frappé de ce que vous avez dit sur les scientifiques. Le respect pour les scientifiques de la part de n'importe qui ne me surprendrait pas tant que de votre part à vous, qui, justement, avez cette singularité d'être un scientifique et de n'avoir pas utilisé les éléments scientifiques pour cela.

CLS : Les sciences humaines ne sont pas les sciences, n'est-ce pas! Si on pouvait avoir la recherche fondamentale et pas la science appliquée, ce serait parfait!

$\mathrm{PM}$ : Est-ce que c'est concevable, une cité scientifique qui serait tout à fait divorcée de la cité tout court?

CLS : Non, ce n'est pas concevable... Ce serait idéal et puis, surtout, il y a des échanges constants entre la science théorique et la science appliquée et je ne pense pas qu'il pourrait y avoir une science fondamentale s'il n'y avait pas à côté une science appliquée.

PB: Quelle place avez-vous fait dans vos travaux et dans vos recherches aux applications, en tout cas scientifiques, d'ordinateurs...?

CLS : Je ne m'en suis jamais servi. J'ai été très stimulé par la lecture des cybernéticiens... J'ai des collègues qui travaillent sur les mythes avec des ordinateurs... Il en sortira des choses probablement plus curieuses que celles que j'ai faites moi-même. On ne sait pas encore comment s'y prendre pour y arriver. Pas encore. Cela dit, je crois que Françoise Héritier est en train de traquer les systèmes crow/ omaha avec un ordinateur. 
PM: Pour en revenir aux Structures... Depuis une vingtaine d'années, on découvre un nombre croissant de systèmes qui ne sont pas unilinéaires (ni patrilinéaire, ni matrilinéaire) et qui, pourtant, ont des choses qui ressemblent à des structures d'échange. La théorie standard semble, devant un certain nombre de faits croissants, inadéquate.

CLS : Dans les limites des systèmes unilinéaires, j'espère avoir mis un peu d'ordre là où il y avait du bazar. De même qu'il y avait eu une tendance à ramener tous les problèmes dans le « corset » unilinéaire, je me demande s'il n'y a pas eu une tendance vers l'autre côté, de façon excessive. [...] Cela n'empêche qu'il y a une masse de systèmes qui sont indifférenciés et qu'il faut arriver à en faire la théorie de façon satisfaisante. À supposer que j'aie liquidé la question de l'unilinéaire, il faut qu'il y ait quelqu'un d'autre (ou plusieurs) qui, maintenant, la liquide de même pour les systèmes indifférenciés. Il faut essayer de rassembler une masse énorme de documentation, et faire la synthèse des deux, qui construira le méta-système. II y a pas mal de questions de mode. On est devant des données contradictoires. L'interprétation en fonction de l'indifférenciation ne permet pas de faire abstraction des faits contraires qui existent.

PM: Même dans les systèmes que vous pensez avoir résolus, des recherches récentes montrent qu'il y a encore des problèmes. Je pense au système australien. La jeune école australienne a proposé un modèle de son système qui est radicalement opposé au vôtre et qui, d'une certaine manière, le remet en cause [...]. On aboutit en ce moment à avoir deux modèles : un modèle qui est un modèle normatif auquel les jeunes ethnologues australiens disent: "Ceci peut réglementer ce qui se passe dans la tête des Australiens, leur monde idéal de la société; mais nous, nous proposons un modèle qui, lui, rend compte de la circulation des femmes, de la réalité des faits d'échange. » A-t-on affaire à deux modèles incompatibles? Est-ce qu'on peut dire qu'un modèle infirme l'autre?

CLS : Je n'ai jamais essayé de chercher autre chose que ce qui se passait dans la tête des gens, et la façon dont ils pensaient leur propre système. Je ne me suis jamais préoccupé de la façon dont ce système fonctionnait réellement dans les faits.

$\mathrm{PM}$ : Je ne suis pas tout à fait d'accord avec vous là-dessus. Un système d'échange, qu'il soit restreint ou généralisé, a des implications sociologiques, au niveau de la façon dont la solidarité sociale en fin de compte s'organise, la façon dont les groupes sont solidaires.

CLS : Ce sont des modèles de laboratoire. J'ai tout à fait pensé les Structures... dans une perspective marxiste, de la façon dont Marx s'y est pris, qui consiste à transporter des faits en laboratoire, et à bâtir des modèles et à voir ce qui se passerait si rien de l'extérieur ne venait infléchir ou modifier le fonctionnement du modèle. C'est très intéressant, mais ça ne suffit pas.

PM: Si les Australiens avaient raison, si les modèles gérontocratiques de mariages australiens fonctionnaient réellement, on pourrait se poser la question de savoir comment il est possible qu'ils aient conçu ce système qui ne peut pas fonctionner dans la réalité, et qui serait donc un mythe. Pourtant, les données sur lesquelles ils s'appuient ne semblent pas être uniquement le résultat des procès de transformation aborigènes.

CLS : Nous avons jusqu'à présent porté une attention principale aux normes et aux faits. 
PM: Quel rapport peut-on concevoir entre ce système de normes et ce système de fonctionnement?

CLS : On peut très bien concevoir de dégager les notions sous-jacentes et les paradigmes qui orientent la pensée juridique française, et puis regarder ensuite comment ça fonctionne dans la réalité. Vous verrez que c'est très différent. Les gens trichent avec la juridiction.

PM: Je pense à toute l'ethnologie africaniste traditionnelle et même récente; on n'y trouve pas toute cette discordance entre les modèles normatifs (que vous avez proposés notamment pour les Australiens) et les modèles qu'on propose actuellement (depuis une quinzaine d'années).

CLS : Je n'ai pas proposé de modèles normatifs, je n'ai rien inventé, puisque les gens avaient observé et j'ai simplement essayé de les classer. J'ai repris les faits de Radcliffe-Brown et de pas mal d'autres, je les ai classés d'une façon un peu différente.

PM: Vous avez fait plus que les classer; vous avez montré leurs principes et leur logique.

CLS : Quand je lis chez les jeunes Australiens - je ne dis pas qu'ils le disent en propres termes - que, depuis Spencer et Gillen, les gens ont complètement rêvé, je ne peux pas le croire.

PM: Je suis d'accord avec vous - ça ne peut pas être un pur fantasme libre et gratuit. Cependant le monopole des jeunes femmes dans les sociétés sur lesquelles on a un minimum de données historiques - je pense aux Tiwi par exemple ${ }^{7}$ - existe bien. La gérontocratie et la monopolisation des jeunes épouses par les hommes les plus âgés semblent être des faits anciens et avérés. Pour beaucoup d'ethnologues (je me mets dans le nombre) et pour tous les Anglo-Saxons, ce qu'ont représenté principalement les Structures..., c'est une classification, mais aussi un principe d'ordre général dans toutes les familles de sociétés. Mais ce principe d'ordre ne s'appliquerait pas seulement à des conceptions. Il s'appliquait à un fonctionnement réel, c'est-à-dire à un échange réel de partenaires et la façon dont les Anglo-Saxons ont interprété les systèmes qu'ils appellent maintenant prescriptifs, c'est bien des normes qui régissent effectivement un échange réel. Vous me dites maintenant que vous vous êtes préoccupé d'une expérience de laboratoire sur ce qui se passait dans la tête des gens, cependant vous faites très souvent référence dans votre livre, et à juste titre, à des mécanismes de solidarité globale.

CLS : Mais naturellement, car on fait un modèle de laboratoire et puis, dans la mesure où les informations dont on dispose le permettent, on vérifie le fonctionnement du modèle sur l'expérience.

PM: Contrairement à ce que disent les Anglo-Saxons, je pense que c'est vérifié beaucoup plus souvent que les critiques les plus pointilleux de votre œuvre ne l'ont fait. Je pense à Needham par exemple, qui réduirait les systèmes prescriptifs par le

7. Charles W. M. Hart et Arnold R. Pilling, The Tiwi of North Australia, Holt, Rinehart and Winston, New York, 1960. 
monde. Ça permet de vous poser une question: ce n'est pas seulement au niveau des normes idéales, vous parlez aussi du fonctionnement réel?

CLS : Disons que ça représente un état d'équilibre du système. Mais, bien sûr, un état d'équilibre avec lequel les gens trichent tout le temps. Je n'appellerai même pas ça des déviances, parce que ce sont des déviances normales.

PM: Il y a une autre objection qu'on peut vous faire, [celle] de Norbert Wiener disant que si l'on prend un système quelconque et si l'on envisage la chose sous l'angle le plus général, qu'on lui donne un programme tout à fait aléatoire, ce système aura différentes positions et il $\mathrm{y}$ en a qui seront plus fréquentes. On conviendra d'appeler ces positions « positions d'équilibre ». Elles nous donneront l'apparence d'une intentionalité et pourront donc nous paraître comme des structures.

CLS : Ce n'est pas recevable. La littérature ethnologique est remplie de preuves que les gens pensent leur système et que l'invention est vraiment dans leur tête. Je ne dis pas toujours et partout...

PM: Vous croyez qu'en fin de compte, le pouvoir régulateur de ces conceptions peut se réaliser dans certaines situations d'équilibre des sociétés et contribuer à cet équilibre.

CLS : Disons que ça a une fonction pilote pour le système.

PM: Ce qui, du même coup, rend toute transformation du système presque incompréhensible?

PB: Dans quelle mesure y a-t-il des exemples, dans la littérature ethnologique, de transpositions de systèmes, de mouvements? Quand on pense que notre société, soit à Lisbonne hier, soit en Grèce avant-hier, peut actuellement changer une mentalité, est-ce que dans les mentalités plus...

CLS : Bien sûr que ça change, selon les auteurs. Stanner a publié des documents merveilleux sur les gens qui s'en vont chez des voisins, qui voient ce qui se passe chez les voisins, et qui disent que c'est beaucoup mieux chez eux ${ }^{8}$.

JMB : Vous pensez que ça suffit à changer la fabrique de tout le système social?

CLS : Il n'y a pas que cela. Il y a des fluctuations démographiques, des guerres, des ambitions individuelles... [...] N'oubliez pas non plus qu'à côté des jeunes Australiens dont vous parlez, il y a d'autres Australiens qui travaillent dans un sens tout à fait différent. Par exemple les articles de Stanner qui restent dans la perspective la plus classique tout en l'enrichissant considérablement.

JMB : La question plus générale qu'on peut se poser est: est-ce que vous n'avez pas contribué à divorcer l'ethnologie d'une sociologie? Au sens classique, au sens durkheimien? C'est-à-dire, en fin de compte, à restreindre l'étude des structures de la parenté à des structures mentales.

CLS : Oui, c'était déjà chez Durkheim. Il n'y a pas que la conscience collective chez lui, mais aussi la solidarité. Mais je n'ai jamais été convaincu de cela.

8. William Stanner, « Aboriginal territorial organization: estate, range, domain and regime », Oceania, 36 (1), 1965, p. 1-26. 
JMB : Pourtant quand vous parlez de l'efficacité respective de l'échange restreint et de l'échange généralisé, on sent comme un écho à la conception durkheimienne de la solidarité.

CLS : On l'a écrit. On a dit que c'était la différence entre la solidarité mécanique et la solidarité organique, mais ce n'est pas vrai du tout. Tout ça, ce sont différentes modalités de solidarité mécanique [...]. Je suis d'accord que le grand challenge de maintenant, c'est le système indifférencié. Mais je pense qu'on arrivera à le traiter de la même façon et dans le même esprit, non pas par rapport à un repère fixe, mais par rapport à des repères qui se redéfinissent eux-mêmes à chaque instant.

JMB : Et dans un système de transformation analogue à ce que vous découvrez à l'œuvre dans les Mythologiques?

CLS : Disons qu'à chaque moment de son devenir une certaine configuration se définit à l'intérieur du groupe social, par rapport à laquelle les choses peuvent se reformuler de la même façon, et non pas un repère fixe qui est donné une fois pour toutes comme la filiation patrilinéaire ou matrilinéaire. Cela se rejoue constamment comme un kaléidoscope où les figures sont constamment différentes et imprévisibles, mais où chaque figure qui se réalise a son plan de symétries, a son centre de gravité...

PM: La préoccupation d'un ordre social ne vous gêne pas?

JMB : On vous a beaucoup reproché de faire du « mentalisme »- l'ordre des structures, vous le définissez principalement aujourd'hui comme étant un ordre mental. L'ordre des sociétés sur lequel on peut se poser des questions est pour vous un phénomène en partie aléatoire? Qui dépend de contingences historiques, d'événements?

CLS : Oui. Je vais user d'une parabole dont je me suis souvent déjà servi. Si vous prenez un coquillage, il y a deux choses: la coquille et le mollusque qui sécrète la coquille. Moi, je fais de la malacologie - je comprends très bien qu'il y ait des gens qui s'intéressent à la façon dont les animaux eux-mêmes vivent ensemble, se disputent, cherchent leur nourriture... Je n'ai absolument pas besoin de cela pour comprendre les fonctions algébriques auxquelles chaque involution des stries de chaque type de coquillage correspond. Ce qui n'empêche pas qu'il y ait un problème très important qui est de savoir pourquoi tel organisme sécrète telle sorte de coquillage et tel autre organisme telle autre espèce.

PM: C'est-à-dire que les formes vous préoccupent plus que l'élément engendreur, ou générateur?

CLS : Oui, à condition de s'entendre sur le mot « générateur » ou « engendreur ». Je peux considérer que les formes s'engendrent les unes les autres. Je peux aussi faire comme s'il n'y avait pas d'organisme du tout et considérer que je pouvais passer par transformation d'une forme simple de coquille à une forme plus complexe et qu'en ce sens ces formes de coquilles s'engendrent les unes les autres.

JMB : Ce qui récuse notre question de savoir s'il y avait, dans la réalité des mythes, autre chose que ce que vous en voyez dans les représentations. L'inscription des mythes.

CLS : Quelle relation doit-on établir entre l'ordre de connaissance et de dévoilement des mythes, et l'ordre des processus de transformations qui les constituent? 
Je n'ai jamais dit que c'était une structure commune. Il faut que vous m'expliquiez cela parce que je suis perdu...

JMB : On vous avait posé la question d'engendrement des coquilles les unes par les autres. On pourrait dire que cet ordre d'engendrement est ou dans un réel, ou dans un ordre de déchiffrement pour le lecteur. Est-ce qu'il y a complète adéquation entre votre lecture, ou votre déchiffrement, et l'ordre de génération de ces structures?

CLS : Il y a d'heureuses rencontres, mais cela n'est pas évident. De toute façon, nous n'avons pas de moyens de le vérifier. Il y a des cas où on peut dire - si vous avez deux étapes $\mathrm{A}$ et $\mathrm{B}$, ça n'a pu que se passer de $\mathrm{A}$ à $\mathrm{B}$; ça n'a pas pu se passer de $\mathrm{B}$ à $\mathrm{A}$.

PM: Quels sont les critères de vérifiabilité de cette adéquation?

CLS : Il peut y en avoir deux. Le premier qui est interne, et les corroborations archéologiques et linguistiques.

PM: En prenant toujours l'Amérique comme terrain, est-ce que vous pensez qu'en fin de parcours vous auriez abouti aux mêmes structures communes et au même système de transformation, entre ces structures que vous dégagez des mythes. Il n'est pas absurde d'imaginer que vous soyez parti de l'Amérique du Nord plutôt que du dénicheur d'oiseaux.

CLS : Il y a une quantité prodigieuse de cheminements possibles. Le réseau des mythes est un réseau qui a une connectivité énorme. J'ai suivi un trajet qui est un trajet possible parmi une quantité d'autres, donc j'aurais aussi bien pu en suivre un autre. Je pense qu'il serait apparu les mêmes vérités sur la nature des rapports qui existent entre les mythes - pas nécessairement les mêmes mythes - ou les mêmes, mais envisagés par d'autres interconnexions.

PM: On suppose qu'il y ait donc un référent extérieur, que ça ne soit pas un intercode complètement lisible. Qu'il y ait une sorte d'au-delà des trajets que vous pouvez...

CLS : Le matériel mythique que j'ai utilisé pour les deux Amériques représente à peu près 10 à $20 \%$ du matériel recueilli. Ce matériel recueilli représente probablement $5 \%$ du matériel qui a existé, alors je trouve que la question n'a pas beaucoup de sens, et je suis comme quelqu'un qui a essayé de faire la description d'une langue inconnue, c'est-à-dire, de façon tout à fait provisoire et partielle, la mise en évidence d'un certain nombre des propriétés du système. Il y en a bien sûr des quantités d'autres, que nous ne connaissons pas, que je n'ai pas atteintes et dont nous ne connaîtrons jamais certaines, parce que nous ne possédons qu'une petite partie du matériel.

PM: Dont l'organisation n'a pas de lois?

CLS : Je suis convaincu qu'elle en a, mais comme elles nous échappent... Les Indiens ont raconté des quantités de mythes que personne n'a recueillis. Supposez que vous arrivez sur une planète inconnue et qu'il y ait des gens qui émettent des bruits. Pendant des années, les gens écoutent ces bruits et disent que ce sont des bruits incohérents. Quelqu'un vient, c'est moi, et dit: c'est une langue, et je vais vous montrer pourquoi c'est une langue. Ça ne veut pas dire que je comprends la langue, qu'il connaît les propriétés de la langue. 
PM: Vous faites donc l'hypothèse que les mythes ont plus de sens quand on les étudie dans le réseau qu'ils forment, à l'échelle continentale, que lorsqu'on les étudie ethnographiquement, c'est-à-dire par rapport aux gens qui les ont produits, narrés, écoutés.

CLS : Je proteste. Car, pour tout mythe que j'ai étudié, j'ai commencé par rassembler la totalité des informations ethnographiques dont on disposait. Je l'ai utilisée à bloc. Seulement cela ne suffit pas, parce qu'un mythe n'est pas la propriété particulière d'un petit groupe qui est sourd et aveugle à tous les autres groupes qui l'entourent. Un mythe est - que les gens en soient conscients ou non - une discussion, un dialogue avec les mythes des gens d'à côté. Une fois qu'on a bien utilisé les informations ethnographiques, premier quart indispensable, il reste encore les trois quarts à faire.

$\mathrm{PM}$ : Je ne vois pas bien en quel sens ce réseau de mythes peut donner naissance à une langue nouvelle. Une langue suppose des interlocuteurs, or personne n'est à l'écoute.

CLS : Quand je dis « une langue », je veux dire que ça a un sens, que ça veut dire quelque chose. Les mythes se parlent entre eux, au moyen des hommes. Un mythe ne s'interprète jamais en fonction d'un code particulier (biologique, astronomique, etc.) mais il consiste à mettre en relation plusieurs codes.

PM: Tels que vous les interprétez, les mythes ne disent pas grand-chose. Finalement, les gens qui les écoutent n'en comprennent qu'une partie. L'ensemble des sens à différents niveaux, y compris celui du symbolisme, est plus restreint que celui que vous dégagez par l'étude d'un réseau de mythes.

CLS : Il est probablement plus vaste. Je n'arrive à en dégager qu'une partie. Il y a probablement plus de choses dans un mythe que ce que j'arrive à en sortir. Plus vous élargissez le réseau mythique, moins il signifie. L'explication par les mythes, c'est une grande illusion.

PM: Néanmoins, vous supposez quand même qu'il y a une pauvreté de sens au mythe, ce que beaucoup d'ethnographes ont dit à tort. Beaucoup d'ethnographes ne se sont pas intéressés aux mythes parce que ce sont des histoires incompréhensibles et les Indiens n'ont pas l'air de les comprendre très bien.

CLS : Ils ne les comprennent pas, mais ça fonctionne. Ça fonctionne par un certain nombre de propriétés logiques qui ne sont pas perçues comme telles, mais qui sont perçues par leur résonance sur une multitude de plans simultanés. Si vous voulez, cela fonctionne comme la musique quand nous l'écoutons et qu'elle nous gratifie. Nous ne savons pas exactement pourquoi.

PM: Avec cette différence qu'il y a une chose qu'on peut faire sur les mythes et qu'on ne peut pas faire sur la musique, c'est les commenter.

CLS : C'est là précisément qu'il faudrait relancer la balle; il faudrait commenter la musique comme nous commentons les mythes. C'est ce que j'ai essayé de faire avec le Boléro.

PM: Il me semble que le commentaire du mythe est homogène au mythe, et le commentaire musical a toujours été pour moi une sorte de scandale.

CLS : La matière première du mythe est le langage, et celle de la musique est hétérogène. Je veux bien qu'il y ait là un écart impossible à combler. 
PM: Et comme vous le savez, dans les mythes qu'on recueille, il y a souvent des commentaires inclus - il est souvent très difficile de faire la part de la narration et de la glose. Il y a sans doute des analogies en musique moderne, de glose et de commentaires sur un thème.

JMB : J'aurais voulu revenir sur votre conception de l'histoire. Vous avez très bien montré comment la dimension diachronique des structures, que vous ne niez pas, malgré tous les reproches éternels qui ont été faits au structuralisme, était autre chose que l'histoire. Est-ce que ça vous paraît fécond de revenir sur la conception que vous avez de l'histoire en tant qu'impact sur la vie des sociétés et leur structuration, l'histoire étant quelque chose dont nous ne pouvons pas nous passer parce qu'elle nous met constamment en face de phénomènes irréductibles - irréductibilité de l'événement par rapport à la structure.

CLS : L'histoire recouvre une quantité d'événements dont nous ne pouvons rien dire d'autre, sinon qu'ils se sont produits. Il y a une quantité de phénomènes structuraux qui se déroulent dans la diachronie mais, en plus de cela, il y a des événements qui ne relèvent, eux, d'aucune structure à ce niveau-là.

PB: Vous admettez une structure venant d'un autre ordre de grandeur?

CLS : Il y a des niveaux de réalité qui sont complètement instructuraux quand nous les saisissons à un certain point, et qui le deviennent quand on en saisit un autre. C'est la grande découverte de Boulainvilliers et de Gobineau à sa suite.

PM: Et entre ces systèmes de transformation structuraux et ces événements, n'y a-t-il jamais de relation traçable, à votre avis? Y a-t-il une incidence des seconds sur les premiers?

CLS : Il peut y en avoir, mais on ne peut jamais postuler qu'il y en a obligatoirement. Ça a été une critique que m'a faite Godelier. Il a prétendu voir une contradiction entre des textes où j'affirmais le primat des infrastructures et l'inanité de l'événement. Ce ne serait vrai que si les infrastructures étaient elles-mêmes sujettes à un déterminisme. Ce qui n'est en aucune façon impliqué. Autrement dit, il ne me semblait pas nécessaire que le commodore Perry ${ }^{9}$ aille au Japon en $1853 \ldots$

PM: Est-ce qu'un historien ne vous demanderait pas si ce n'était pas fort probable qu'il y fut allé dans ces dix années-là? Ce n'est pas l'année qui compte, mais le moment... Cela devait se produire.

CLS : Je suis d'accord pour dire que c'était probable, mais pas que c'était nécessaire. Il aurait pu se produire une grande crise économique aux États-Unis ou en Occident, ou il aurait pu y avoir une guerre entre l'Europe et l'Amérique et cela ne se serait pas produit. Une épidémie de peste, ou toute autre chose.

PM: Les historiens semblent croire beaucoup à la nécessité. La découverte de l'Amérique semble être la clôture du chemin pour aller en Chine.

CLS : Ça n'est absolument pas une nécessité. Ça explique et permet de comprendre pourquoi ça s'est passé comme ça. Il aurait pu se passer mille autres choses.

9. Le commodore Perry commandait la flotte américaine qui a été reçue au Japon en 1853, ce qui fut le signal de l'ouverture de ce pays au monde occidental après des siècles d'isolement. 
PM : Pas sûr. Certains écrits vont jusqu'à dire que les premières activités qu'on a installées en Amérique (dont la découverte est sans doute fortuite) étaient du même ordre que ce qu'on voulait faire avec la Chine. C'est-à-dire des matières précieuses.

CLS : Peut-être que je ne sais pas ce que c'est que la nécessité.

PB: Quelle est la position entre la fermeté de la structure et l'irréductibilité de l'événement?

CLS : Supposez que Christophe Colomb ait découvert l'Amérique et que les Aztèques aient eu la peau de Cortez [...]. Une chose m'a beaucoup frappé lorsque j'ai été à Vienne et que j'ai visité le trésor des Habsbourg, où il y a à la fois toutes ces tapisseries, ces objets européens et les trophées mexicains. Supposez qu'ils aient été les plus forts et que cela ait amené les Habsbourg, au lieu de faire la guerre, à s'unir aux Mexicains. Toute l'histoire aurait été complètement changée. Je pense qu'il existe des moyens de comprendre pourquoi les choses qui se sont passées se sont passées. Ça ne veut pas dire qu'elles devaient se passer de cette façon-là.

JMB : C'est très important. Cette irréductibilité de l'événement ne vous fait pas voir en lui davantage qu'un clinamen, au sens des Épicuriens ${ }^{10}$ ?

CLS : Si, sûrement.

JMB : Ces événements, leur production dans le temps, n'a jamais de rapport avec la temporalité dans laquelle les structures se combineraient ou se transformeraient les unes dans les autres: il n'y a jamais pour vous - et ce serait un problème de causalité (métaphysique) -, il n'y aurait jamais, donc, de rapport entre les transformations des structures les unes dans les autres et l'impact de ces clinamens? Est-ce que ces «événements dérangeants » peuvent amener des processus de transformation structuraux?

CLS : Sûrement. Prenez un exemple théorique. Une société qui est organisée en un certain nombre de clans. Survient une épidémie qui détruit la moitié de la population et qui fait que certains clans disparaissent. Il faut que la chose se réorganise, et les clans vont se mettre à former un nouveau système.

JMB : Est-ce qu'il y a incidence de la production de l'événement sur la forme ultérieure du système, ou sur le choix d'un itinéraire de transformation? Étant bien entendu que l'événement n'a pas de nécessité, est-ce qu'il détermine une certaine configuration, par son impact, des structures en aval?

CLS : J'imagine qu'on pourrait trouver des exemples de toutes les combinaisons possibles, de toutes les modalités possibles. On ne peut pas, en tous cas, l'édicter sous forme de principe.

PB: Est-ce qu'il y a pour Claude Lévi-Strauss un ordre établi?

JMB : Non, ce n'est pas tout à fait ça. Je me demandais si on pouvait étendre en sortant de l'aléatoire, dans le système de transformation structurale, l'incidence de

10. Clinamen : terme signifiant « déviation », utilisé notamment par Lucrèce et les atomistes pour se référer à la déviation infime supposée rendre compte de l'existence du monde en permettant aux atomes de se rencontrer au lieu de tomber parallèlement, sans jamais se rencontrer. 
tel ou tel événement en tant que levier ou point d'appui sur la production de telle ou telle structure, dans le champ du possible.

CLS : Je ne crois pas que les ethnologues soient équipés pour répondre à de telles questions. Cela supposerait qu'on puisse monter des expériences dans les laboratoires.

JMB : C'était l'idée de certaines règles de transformation. L'articulation, l'impact de l'événement et les règles de transformations dans l'histoire d'une société, puisque vous avez souligné l'importance de la déviance dans cette histoire.

CLS : Ce qu'on peut dire, c'est que les événements obligent les structures à se transformer; dans certains cas on peut arriver à voir comment.

PM: Les événements qui manifestent une certaine dose de prévisibilité, si on était beaucoup plus équipé : mouvements anomiques dans la tradition durkheimienne, qui a montré que ces conduites individuelles anomiques en fait exprimaient des mouvements de foules, de nature sociale et collective.

CLS : Je ne peux pas penser que les mouvements messianiques sont des mouvements anomiques. Ce sont au contraire des mouvements qui essaient de réorganiser la structure. Ce sont des réorganisations.

PM: Est-ce que vous pensez que ces faits d'anomie sont susceptibles d'être analysés structuralement?

CLS : Par définition, ce sont des faits statistiques. Je veux dire par là que ce sont des conduites qui se manifestent chez une certaine proportion d'individus à l'intérieur du groupe social et que vous les appréhendez par la voie statistique [...]. La statistique en elle-même ne veut rien dire, elle est l'indice de quelque chose. Différence structurale qui explique un écart entre deux sociétés. Les faits d'anomie pourront vous mettre sur la piste d'une propriété structurale, mais en eux-mêmes ne la constituent pas.

PM: Est-ce que les faits comme la sorcellerie, vous les considéreriez comme des faits d'anomie?

CLS : Sûrement pas.

PM: Elle implique pourtant un certain nombre de désordres sociaux.

CLS : Ce ne sont pas des désordres, ce sont certains aspects de l'ordre social dans une société où il y a des sorciers.

PM: C'est aussi un phénomène soumis à une assez grande variabilité historique. En cela, c'est peut-être comparable au taux des suicides.

JMB : Je ne pense pas personnellement que la sorcellerie soit un fait dont les conséquences sont toujours les mêmes. Ça existe comme système dans toutes les sociétés, mais je ne pense pas que les conséquences soient uniformément les mêmes dans le temps, et dans l'espace, bien entendu.

CLS : Vous entrez là dans une zone où vous êtes obligé de faire appel à l'histoire de la société. Je ne pense pas du tout que l'approche structurale oblige à s'intéresser à tout et permette encore moins de tout expliquer. Ce n'est jamais la société qui est considérée comme une totalité... statique. Il s'agit d'expliquer un certain nombre de choses à l'intérieur de la société. 
JMB : On prête à tort à l'explication structurale une ambition d'exhaustivité et on lui reproche ensuite de ne pas l'avoir suivie.

CLS : C'est très juste. Il y a, dans les sociétés humaines, certaines plages où il est possible de faire comme si le nombre de variables était petit. C'est à ça que je m'intéresse. Je ne nie absolument pas qu'il y ait d'autres plages ou d'autres niveaux où les choses se passent différemment.

JMB : Est-ce qu'on n'est pas obligé, ethnologue, de faire une hypothèse provisoire d'ensemble de la société, de la considérer provisoirement comme une totalité? Je ne parle pas de l'ethnographe. Mais est-ce que l'ethnologue, l'analyste ne fait pas la même hypothèse?

CLS : Il est beaucoup plus facile de considérer comme une totalité une société qui est petite en volume et, donc, quand il s'agit de très petites sociétés, on se rapproche de cette limite. Quand il s'agit de sociétés plus nombreuses et plus compliquées, on en reste toujours très loin. Ce n'est absolument pas la même méthode, la même attitude d'esprit, la même attitude épistémologique, qu'on peut avoir devant une tribu brésilienne dont l'effectif est de 300 personnes et devant une société africaine qui en a 30000.

PM : Cela interdirait alors le retour vers une fameuse anthropologie des sociétés complexes...

CLS : Non, quand nous sommes devant ces sociétés complexes, les plages auxquelles nous avons accès par les méthodes structurales sont beaucoup plus petites et moins nombreuses.

PM: Vous admettez qu'il y ait des implications idéologiques aux choix méthodologiques que vous avez faits. Je pense à des prises de position épistémologiques pures qui ont des conséquences quant à une vision de l'histoire, également quant aux rapports entre l'observateur et l'objet...

CLS : On peut faire des prévisions qui tiennent à des rapports de force et qui n'ont rien de structural, et encore...

PM: Est-ce qu'au contact des sociétés (les nôtres), je pense à l'Amérique, les formes culturelles développées [résultant] d'interférence sont susceptibles d'analyses aussi fécondes et aussi fines que celles faites sur des sociétés davantage isolées? Et est-ce que les phénomènes de greffe de certains mythes ou de certaines idéologies ou certaines distorsions sont susceptibles d'un renouvellement de l'approche ethnologique? Je pense à la contagion par notre société de sociétés d'Indiens qui changent les contenus et les formes de leur culture - éclatement de la topologie d'un village par suite de l'agression des missionnaires [comme chez les Bororo]...

CLS : Les Colombiens [Amérindiens de la Colombie britannique] sont en train de recélébrer des rituels traditionnels qui n'avaient sans doute pas disparu et se faisaient clandestinement et qui maintenant se font plus « librement » et ne sont plus passibles de persécutions. On aurait pu supposer que, sous l'impact missionnaire ou autre, ces rituels disparaissent complètement - c'est vrai de certains d'entre eux... Or ce qui se passe actuellement, c'est qu'ils se poursuivent avec un changement de finalité, et l'initiation se trouve ainsi avoir des fins thérapeutiques (boisson, vol...). Personne n'aurait pu prévoir cela. 
PM: Est-ce que cette fonction «salutaire » ne faisait pas partie de l'initiation traditionnelle multifonctionnelle?

CLS : Comme ethnologue, ce qui m'intéresse passionnément est que ça m'aide à mieux comprendre ce qui est consigné dans la littérature, grâce à ce qu'il en subsiste. Bien entendu, un sociologue ou un psychologue y cherchera tout autre chose.

JMB : Dans notre propre société, l'étude de villages, de sociétés rurales, assez closes... relève-t-elle d'une approche ethnologique comparable à celle qui vous a porté vers les rivages de l'autre...

CLS : Il y a une quantité de choses que le sociologue, le psychologue, l'historien, ne peuvent pas voir, et que l'ethnologue peut voir parce qu'il a d'autres techniques et d'autres méthodes d'approche. Nous disposons d'une gamme d'expériences humaines et donc nous pouvons trouver ailleurs des choses qui nous aident à mieux comprendre. Nous ne travaillons pas avec des livres ou des statistiques, mais nous essayons de suivre la vie et la pensée des gens au jour le jour et cela permet « d'atteindre » des choses qui ne sont pas accessibles d'autre façon.

JMB : Et le fait que ces sociétés "prochaines » aient évolué selon le rythme d'une histoire qui est la nôtre affecte-t-il ce regard ethnologique qu'il est possible d'y porter?

CLS : De même que, lorsqu'on veut étudier structuralement quelque chose, il faut commencer par ramasser toute l'ethnographie disponible, de même pour une société contemporaine, l'ethnographie n'a pas qu'une dimension; elle en a deux: spatiale et temporelle. Ce que nous apporte l'histoire est un matériel ethnographique.

JMB : L'histoire nous apporte plus, des encombrements - une notion implicite de la causalité séquentielle par simple succession. Il y a une tendance à considérer que l'événement précédent a en partie provoqué l'événement qui succède.

CLS : Il aide à le comprendre, à l'expliquer, je ne dirai pas qu'il l'a provoqué, parce qu'il aurait pu en provoquer un autre.

JMB : Vous vous intéressez à des masques actuellement, avec une problématique... Comment arrivez-vous à faire l'économie - à court-circuiter - de tout le regard esthétique que notre société porte sur ces masques, pour les analyser comme des objets signifiants ethnologiquement?

CLS : En considérant les masques non pas en eux-mêmes, mais dans les rapports qui les unissent. Sur le plan purement plastique, le rapport est rigoureusement identique à celui qui existe entre les mythes respectivement attachés aux masques en question. Si le rite A contredit le rite B, le mythe A contredit le mythe B et, plastiquement, le masque A contredit le masque B [...]. Mais les gens n'ont absolument pas la notion de ce rapport. Ils considèrent qu'ils ont la propriété exclusive d'un type, donc l'autre type n'existe pas pour eux.

PB: Est-ce que la plastique ou l'expression a une signification en dehors de celle que vous rejetez pour faire une analyse plus rigoureuse et plus profonde?

CLS : Dans certains cas, la plastique s'accompagne de certaines intentions et de certaines significations; dans d'autres, elle est purement traditionnelle. Le masque est comme ça parce qu'il doit être comme ça. Les masques sont tout de même 
beaux pour les gens qui les utilisent. En Afrique, on a essayé de voir la manière dont les usagers eux-mêmes jugeaient de la qualité des masques faits par des sculpteurs différents.

JMB: La fabrication du masque, qui est un acte important, pas forcément entouré de rituel, est-elle liée chez l'artisan à la possession d'un certain type de savoir particulier?

CLS : Elle est liée à la possession, au droit de faire le masque justifié par la légende, à un certain nombre de rites de sa lignée ou de sa place sociale. Il le fera en s'inspirant d'un modèle plus ancien.

JMB : Est-ce qu'il a un savoir de ce mythe dont il illustre...

CLS : J'ai rencontré un chef indien qui m'a longuement raconté le mythe en vertu duquel sa famille est propriétaire d'un certain nombre de masques. Il m'a dit qu'il avait l'intention d'en resculpter un et de recélébrer le rituel. Il y a beaucoup de types de masques...

JMB : L'artisan a donc le droit de faire le masque. Il n'utilise que son savoir-faire, ne met pas en jeu dans sa fabrication un autre savoir?

CLS : Dans ce cas particulier, non, mais il y en a d'autres.

JMB : Y a-t-il conscience d'une symbolisation possible entre les instruments iconographiques abstraits, les ornements du masque et les éléments signifiants dans le mythe?

CLS : Le mythe décrit le masque, ou l'inverse.

PB : À partir du moment où il n'y a pas de savoir esthétique, ni aujourd'hui, ni il y a cent ans, il est évident que ce non-savoir produit le même objet...

JMB : Dans le cadre de l'unicité de l'objet d'art, nous savons que, dans certaines sociétés, un objet a une valeur telle qu'on le garde, et d'autres où des objets fort beaux sont immédiatement jetés une fois le rituel terminé. Est-ce que vous pensez que l'on peut relier ces attitudes à quelque chose?

CLS : Il y a des intermédiaires. Il y a des objets qu'on garde pendant une période déterminée rituellement et après laquelle ils sont détruits. Il y a aussi des peuples qui ont gardé des trésors et les ont fétichisés. Les Indiens d'Alaska ont refusé récemment une proposition de 400000 dollars pour l'acquisition de quelques sculptures - et ce sont des gens très pauvres.

PM: Est-ce que vous croyez qu'on peut relier cela à un mode de permanence sociale qui serait fondé sur l'unifiliation? Au fur et à mesure que j'avance dans mon exploration de l'Amérique du Sud, j'ai l'impression qu'il se confirme, à presque chaque lecture, que c'est une région du monde où le mode de permanence sociale n'est pas fondamentalement organisé par la filiation, la transmission d'un nombre d'éléments de père en fils. Si vous prenez le cannibalisme, on est frappé par une sorte d'horizontalité. Or toutes ces sociétés n'ont pas de trésors.

$\mathrm{PB}$ : En poussant le raisonnement jusqu'à l'absurde, on pourrait dire que, sans conserver ses traces, une société ne peut pas s'organiser et s'établir?

CLS : Ça dépend. Chez les Bororo, qui fabriquent pour leurs fêtes un tas d'ornements, certains sont jetés et d'autres conservés précieusement et transmis de génération en génération. 
Philippe ErIKson, avec la collaboration de Dominique Michelet

JMB : Est-ce que ce qui compte le plus n'est pas la relation qu'ils entretiennent avec cet esprit totémique originaire, donc l'objet devient secondaire?

CLS : Il y a aussi des objets périssables et d'autres moins, ou non périssables.

PM: Il y a des objets non périssables réutilisés de façon constante, mais dont la fonction est variable. Les haches de pierre, par exemple. Souvent, brisées, elles sont réutilisées comme amulettes ou objets magiques. La réutilisation et la conservation sont pour moi très opposées.

CLS : Il y a des objets d'origine humaine et des objets d'origine surnaturelle. Il y a des objets qui sont des preuves, des témoignages, d'autres qui n'en sont pas. 